\title{
QUANTITATIVE TWO-DIMENSIONAL CLASSIFICATIONS OF LOW DISPERSION OBJECTIVE PRISM SPECTRA
}

\author{
Th. Schmidt-Kaler
}

Astronomisches Institut Bochum

The objective prism (or grating) is the most efficient stellar spectrograph since it suffers no light losses at a slit and - compared to a slit spectrograph - only small losses in the camera optics. However, objective prism spectra have two principal disadvantages:

(1) the spectral resolution (and consequently the sharpness of spectral features) depends on the seeing, which may vary from plate to plate.

(2) quantitative measurements are difficult because of the difficulty in obtaining an exact photographic calibration; no comparison spectrum can be placed near the stellar spectra.

Therefore, low dispersion objective prism spectra have generally been used only for very rough estimates of the spectral types and for surveys for certain natural groups like the OB-stars.

In the past years Schmidt-Kaler et al. (1976) have developed methods to determine accurate, quantitative, two-dimensional classifications from objective prism spectra. We circumvented the first difficulty by restriction to equivalent widths (or similar quantities) which are - as a measure of energy - in principle independent of the variable instrumental profile. We overcame the second difficulty by constructing the characteristic curve of the plate directly from measurements of spectra on it near $4400 \AA$ (and $3500 \AA$ ) and photoelectrically measured intensities of the same stars in the B (and U) band of the UBV system. Corrections to the broad band magnitude $B$ (and $U$ ) have been computed to obtain the monochromatic magnitude near 4400 \& for

29 
various spectral types and reddenings; these corrections are 0.02 or less if the photoelectric calibration sequence is restricted to $0-F 5$ stars with $E_{B-V} \leqslant I^{m}$.

The line-free continuum was determined by inspection of high-dispersion spectral atlasses. In the range 0-F5 we found for the dispersion $570 \AA / \mathrm{mm}$ of the Hamburg Schmidt plates 9 useful windows in the blue-UV region. In the case of later type stars a pseudo-continuum was defined and an equivalent area (= line depth $\mathrm{x}$ half width) replaced the equivalent widths when the wings of the lines were heavily disturbed by blends.

For a realistic test of the method we took three OB-associations on plates of the Hamburg-Cleveland survey. The internal s.e. of the equivalent widths of the Balmer lines $\mathrm{H}_{\boldsymbol{\gamma}}$ and $\mathrm{H}_{\boldsymbol{\sigma}}$ was about $1.5 \AA$ (I plate) or more exactly, for $\mathrm{H}=\frac{1}{2}\left(\mathrm{H}_{\boldsymbol{\gamma}}+\mathrm{H}_{\mathbf{\delta}}\right)$,
$\varepsilon\left(\mathrm{W}_{\mathrm{H}}\right)=0.7+0.07 \mathrm{~W}_{\mathrm{H}}[\AA]$.

This is confirmed by comparisons with results from slit spectra, and with photoelectric measurements of $\mathrm{H} \beta$.

Measures of the Balmer jump and color-indices were determined with an error of about $\pm 0 \mathrm{~m}_{0} 3$ ( 1 plate). On the basis of these measurements (basically the $\mathrm{D} / \mathrm{W}_{\mathrm{H}}$ diagram as in the Paris system and/or $(\mathrm{U}-\mathrm{B})_{\mathrm{O}} / \mathrm{W}_{\mathrm{H}}$ ) it was possible to classify the stars in the domain BO-F2 quantitatively in two dimensions with about the same accuracy as the MK-system. This statement is based on 194 stars classified in the MK-system (Fig. 1). At even lower dispersion we could use two plates taken with the thin prism at the Michigan Schmidt $(1400 \AA / \mathrm{mm})$ at CTIO which Dr. Duerbeck kindly put at our disposal.

The internal accuracy of the Balmer line equivalent widths was $1.6 \AA$ and the Balmer jump turned out to be almost the same so that about the same classification accuracy is expected for BO-F2 stars. However, for a check only HD spectral types were known in sufficient number.

Considering the tremendous number of spectra on a typical Schmidt plate it is obvious that the procedure had to be automatized. For this purpose the microphotometer has been connected to an HP $2114 \mathrm{~B}$ computer, and measurements, reductions and classification processes have been completely computerized. The total time for the classification of one spectrum is $2.5 \mathrm{~min}$ including visual controls, and $30 \mathrm{sec}$ without these.

The collaboration of $\mathrm{H}$. Unger is gratefully acknowledged. 


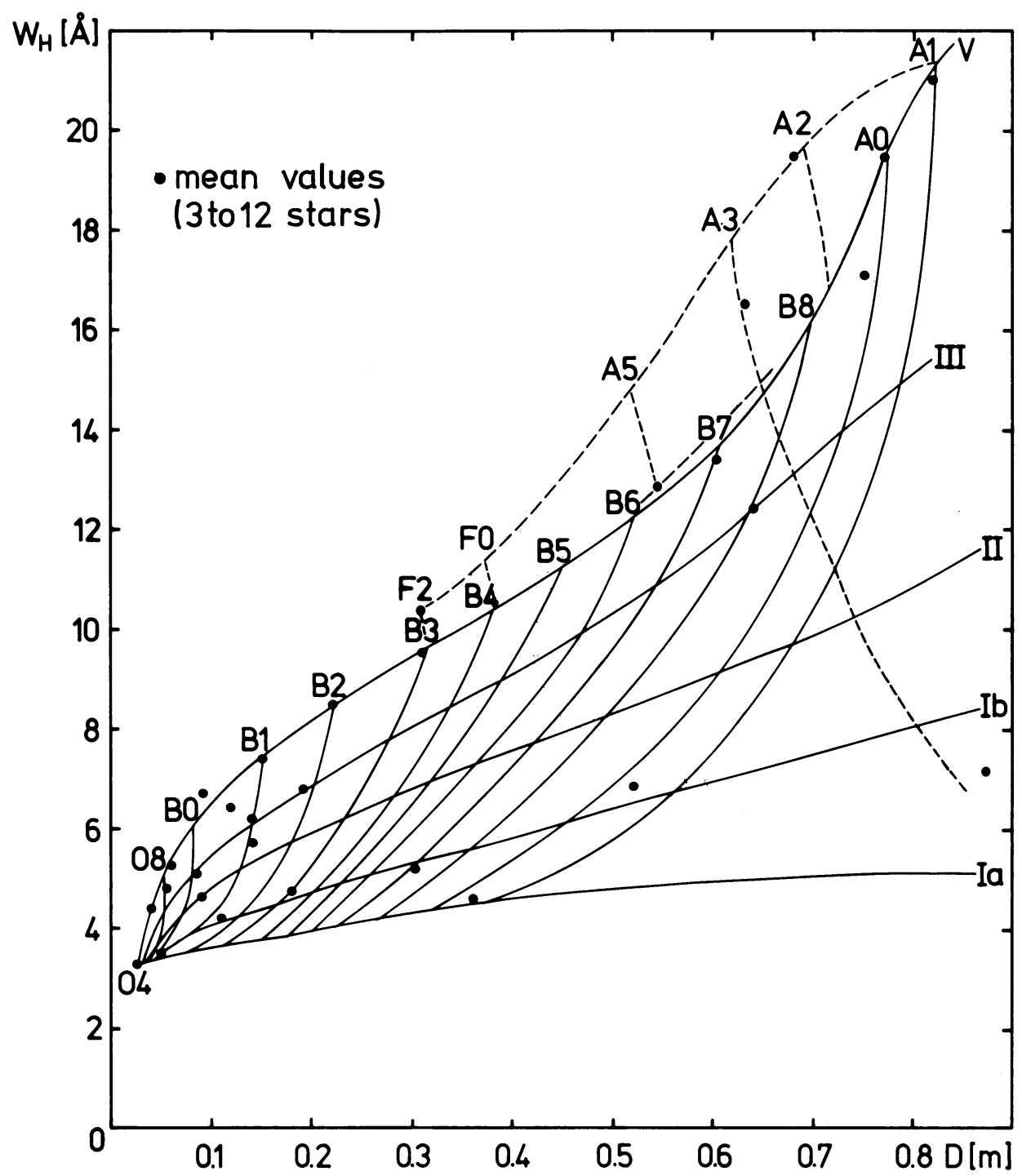

Fig. 1. Two-dimensional spectral classification of early-type stars by Balmer jump (D) and Balmer line intensity $\left(W_{H}\right)$ from Hamburg Schmidt plates.

\section{REFERENCES}

Schmidt-Kaler, Th., Diaz-Santanilla, G., Rudolph, R. and Unger, H. (1976). Quantitative Klassifikation von Sternspektren mittels Objektivprismenaufnahmen. Forschungsberichte des Landes Nordrhein-Westfalen Nr. 2595, Westdeutscher Verlag, Opladen. 


\section{DISCUSSION}

CAYREL de STROBEL: To what apparent magnitude have you observed?

SCHMIDT-KALER: The limiting magnitude of the Hamburg Schmidt survey plates is about $13^{\mathrm{m}}$. With longer exposure and less widening you can certainly reach $15^{\mathrm{m}}$. With the Michigan Schmidt (very low dispersion) on Cerro Tololo (very good seeing) you can reach $16^{\mathrm{m}}$ or $17^{\mathrm{m}}$. The most serious problem is overlap of spectra in crowded areas. 